\title{
Hierarchy Analysis Method for Management Information Systems
}

\author{
Xiao-xia Wang, Yuan Ling \\ Beijing Jiaotong University, School of Traffic and Transportation \\ wangxiaoxia@jtys.bjtu.edu.cn
}

\begin{abstract}
There are many kinds of management information systems (MISs) whether in industries or in enterprises. In China, most of MISs were put into application at different time and with different demands. With the broader application of MISs, it's becoming greater important to integrate them and realize information sharing. Hierarchy analysis method gives out a general structure for the complicated integration of MISs. There are two steps for this method. (1)measure the information communication relations among MISs,(2) divide MISs into multi grade structure. For China railway industry, there are 13 kinds of big MISs, take them as an example to illustrate how to apply this method. Based on hierarchy analysis, MISs with strong connections have priority while integrating MISs. The higher the grade of MIS, the greater overall layout design priority should be given, for more information from other MISs finally reach it.
\end{abstract}

\section{Introduction}

China railway informationization begins early and develops in a rapid pace. In 1980s, computers were widely used in every department of railway; in 1990s, railway information technology application developed into systematization, and management information systems (MISs) for every function have been set up, including railway transport, vehicle, machine, engineering, electricity, finance, statistics and office. The real-time network system of railway transport and production was herein formed.

Experts reached a common sense on China railway informationization, which was that a rudiment frame appeared and it played a rather important role to raise the quality and capability of railway transportation and insure security of transportation. However, because of the gradual establishment of MISs, the information resources sharing is not sufficient, and the exploitation and utilization ratio is relatively low. In view of the overseas railway industry development, there is a great gap in the fields of operation and management, electronic commerce, intelligent transportation, etc.

Please use the following format when citing this chapter:

Wang, X., I.ing, Y., 2007, in IFIP International Federation for Information Processing, Volume 25I, Integration and Innovation Orient to F-Society Volume1, Wang, W. (Eds), (Boston: Springer), pp. 507-5t4. 
On one hand, these problems are caused by the cosmically introduction of MISs at the very beginning of railway informationization; on the other hand, the original layout of railway MISs was based on the theory of traditional administration levels and function division, which was relatively low in view of the prediction level.

\subsection{Known Results}

From the aspect of economy management, there are many methods applied to MISs planning, such as critical success factors, strategy set transformation, business system planning, business information analysis and integration technology, reclaim of investment, and so on[1]. These methods were equal to apply to the micro plan of MISs in the enterprises. When they were applied to industries, such as railway, it would be too comprehensive to make an overall plan. In the fields of database, traffic and transportation plan, there also was a planning method named as cell group ranking, with the characteristic of the evident hierarchy and generally fining function. However it was rather simple from another point of view.

\subsection{Our Results}

Based on the informationization fruits of China railway in the past 40 years, the communication relationship among every big railway MISs are measured by applying hierarchy analysis method of general structure, and divide them into multi hierarchy structure. It resolves the problem that the emphasis of China railway informationization is not obvious, which caused by the simple enumeration of MISs, and provides decision-making support for the great-leap-forward development task of China railway informationization.

\section{Method of Hierarchy Analysis of General Structure}

\subsection{Problem Description}

Hierarchy analysis of general structure analyzes the frame of comprehensive MISs by the theory of relating matrix in gragh theory, making sure the relation among the elements within the system, and divides the comprehensive system into multi hierarchy structure. This gives out qualitative analysis on the potential relations which are not sure under the comprehensive conditions, and provides evidence for rational description[2].

There are two steps for the hierarchy analysis of general structure.

Firstly, establishes a system structure model to describe the relations among the elements within the system. The relations include cause and effect, up and down, affiliation, primary and secondary relation and aim-means relation. The generally describing method is the vector graph, in which the direct and indirect relations among elements could be measured separately by abut matrix and extendable matrix.

Abut matrix $\mathrm{M}$ is a boolean matrix, describing the direct influence among each neighboring elements in the vector graph. The two abut correlated elements in the 
matrix can be defined by two-value relation R. In the matrix, the row element is $S_{i}$, while the line element is $S_{j}$. If there is influence, it is expressed as $S_{i} R S_{j}=1$; if there is no influence, it expressed as $S_{i} R S_{j}=0$. When $S_{i} R S_{j}=1$ and $S_{j} R S_{i}=1$, $S_{i}$ and $S_{j}$ have strong connection.

Extendable matrix $\mathrm{R}$ illustrates to what extent that each nodes can be arrived at by certain routes. It is acquired by abut matrix plus identity matrix $\mathrm{I}$ and several other operations. If $(M+I)^{l} \neq(M+I)^{2} \neq(M+I)^{3} \neq \cdots \neq(M+I)^{r-1}=(M+I)^{r}$, then extendable matrix $R=(M+I)^{r-1}$.

In matrix $\mathrm{R}$, the element $r_{i j}=1$ means that there is at most ( $\mathrm{r}-1$ ) routes reaching this node. $R$ is also a boolean matrix, and the operations are all boolean operation which is $0+0=0,0+1=1,1+1=1,0 \times 0=0,1 \times 0=0,1 \times 1=1$.

Secondly, makes sure the grade of the system structure. Builds extendable matrix $\mathrm{R}$ up into two son-aggregates: (1) for each element $S_{i}$, gathers all related elements that $S_{i}$ may reach into one aggregate, which is called the extendable aggregate $R\left(S_{i}\right)$ of $S_{i}$. (2)Gathers all the elements that may reach $S_{i}$ into another aggregate, named the cause aggregate $A\left(S_{i}\right)$ of $S_{i}$. Along each row, examines each horizontal row of extendable matrix $\mathrm{R}$, all the rows that corresponding the element 1 are in the son-aggregate $R\left(S_{i}\right)$. Then examines each vertical line, all the lines that corresponding the element 1 are in the aggregate $A\left(S_{i}\right)$. In the highest grade of a multi-grade structure, no higher element reaches it, so its extendable aggregate only includes certain strong connection elements of its grade and itself. The cause agrregate $A\left(S_{i}\right)$ of the highest grade elements includes itself and all the elements of extendable lower grade. The qualification for $S_{i}$ is the highest grade element is that $R\left(S_{i}\right) \cap A\left(S_{i}\right)=R\left(S_{i}\right)$. Knows the highest grade elements and scores them out of the table, then the each element of the lower grade gets, until scores out each element by the grade. Finally, the multi hierarchy structure chart can be drawn according to the result of grading, which conduces to the understanding of the grading relation and detail structure of the system.

\section{Operation and Application of Method}

There are 13 big MISs for railway daily operation, namely security MIS, infrastructure establishment MIS, planning MIS, personnel MIS, labor and capital MIS, statistics MIS, vehicle MIS, electricity affair MIS, power and water supply MIS, machine MIS, engineering affair MIS, finance and account MIS, transportation MIS. Takes their information communication relations as an example, expatiates the 
operating steps and conclusion analysis of the hierarchy analysis method of general structure.

Firstly, examines the information communication relations in every railway management function, draws the vector graph of the information communication between each MIS (Fig.1). The number 1 to 13 individually stands for security, infrastructure establishment, planning, personnel, labor and capital, statistics, vehicle, electricity affair, power and water supply, machine, engineering affair, finance and account, transportation MIS.

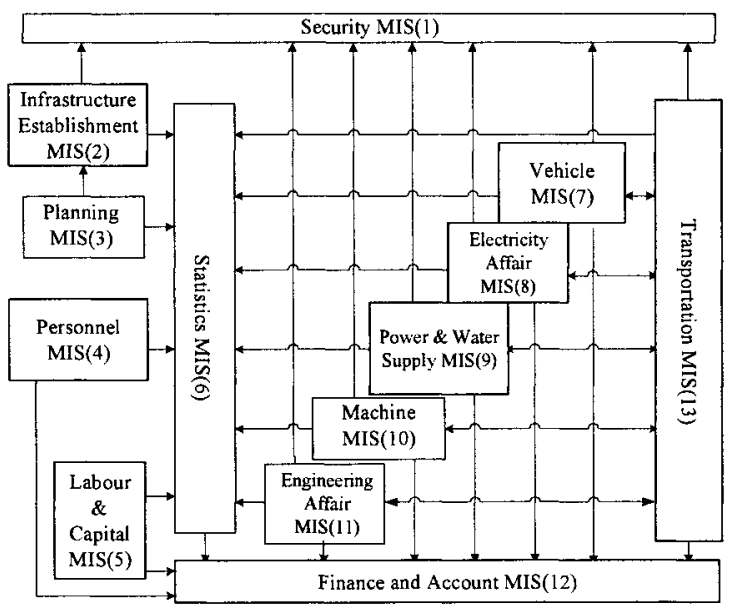

Fig. 1. The vector graph of the information communication in each MIS

In Fig.1, there exist direct and indirect influences among each MIS. For $(M+I)^{2}=(M+I)^{3}$, the extendable matrix is $R=(M+I)^{2}$. The abut matrix $\mathrm{M}$ and extendable matrix $\mathrm{R}$ are as follows.

The symbol "*" in extendable matrix $\mathrm{R}$ does not exist in the original matrix $(\mathrm{M}+\mathrm{I})$. It reflects the indirect relation among elements.

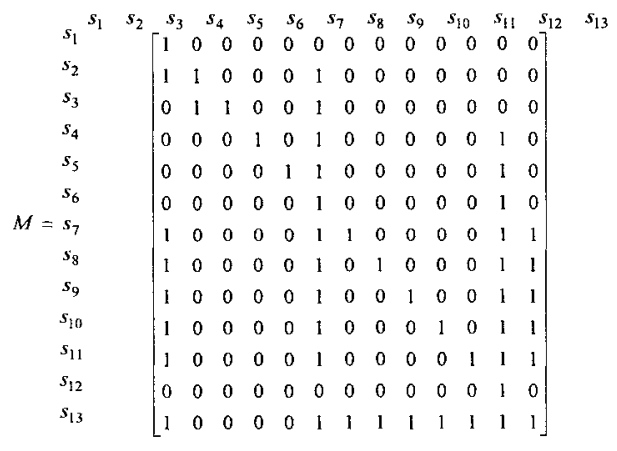




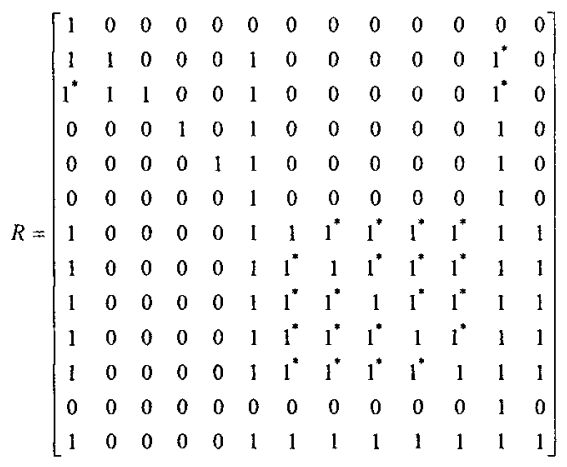

Secondly, makes sure the class of the system structure. The $R\left(S_{i}\right), A\left(S_{i}\right)$ and $R\left(S_{i}\right) \cap A\left(S_{i}\right)$ of this example are shown in Tab.1.

Table 1. The Extendable Aggregation and Reason Aggregation of Extendable Matrix R

\begin{tabular}{|c|l|l|l|}
\hline$S_{i}$ & \multicolumn{1}{|c|}{$R\left(S_{i}\right)$} & \multicolumn{1}{|c|}{$A\left(S_{i}\right)$} & $R\left(S_{i}\right) \cap A\left(S_{i}\right)$ \\
\hline 1 & 1 & $1,2,3,7,8,9,10,11,13$ & 1 \\
\hline 2 & $1,2,6,12$ & 2,3, & 2 \\
\hline 3 & $1,2,3,6,12$ & 3 & 3 \\
\hline 4 & $4,6,12$ & 4 & 4 \\
\hline 5 & $5,6,12$ & 5 & 5 \\
\hline 6 & 6,12 & $2,3,4,5,6,7,8,9,10,11,13$ & 6 \\
\hline 7 & $1,6,7,8,9,10,11,12,137,8,9,10,11,13$ & $7,8,9,10,11,13$ \\
\hline 8 & $1,6,7,8,9,10,11,12,137,8,9,10,11,13$ & $7,8,9,10,11,13$ \\
\hline 9 & $1,6,7,8,9,10,11,12,13$ & $7,8,9,10,11,13$ & $7,8,9,10,11,13$ \\
\hline 10 & $1,6,7,8,9,10,11,12,13$ & $7,8,9,10,11,13$ & $7,8,9,10,11,13$ \\
\hline 11 & $1,6,7,8,9,10,11,12,137,8,9,10,11,13$ & $7,8,9,10,11,13$ \\
\hline 12 & 12 & $2,3,4,5,6,7,8,9,10,11,12,13$ & 12 \\
\hline 13 & $1,6,7,8,9,10,11,12,137,8,9,10,11,13$ & $7,8,9,10,11,13$ \\
\hline
\end{tabular}

In Tab.1, the system number of the highest grade is 1 and 12 , then score out the 1st and 12th line, and the systems 1 and 12 in other lines. Continually follows the search principle of $R\left(S_{i}\right) \cap A\left(S_{i}\right)=R\left(S_{i}\right)$. Finally, the arrange order of system structure is $S=\{1,12,6,2,4,5,7,8,9,10,11,13,3\}$. According to this order, the matrix is rearranged as follows: 


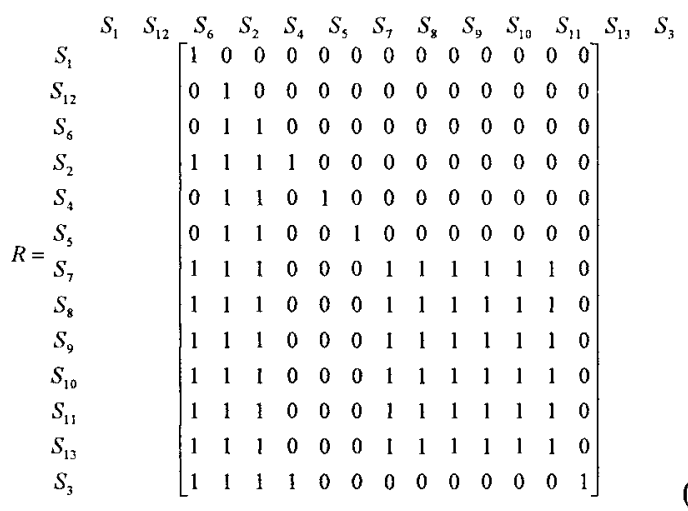

$S_{7}, S_{8}, S_{9}, S_{10}, S_{11}, S_{13}$ forms a son-matrix whose elements are all 1, demonstrating that they have the strong circulation connection.

Finally, draws the multi hierarchy structure of information communication in MISs as Fig.2.

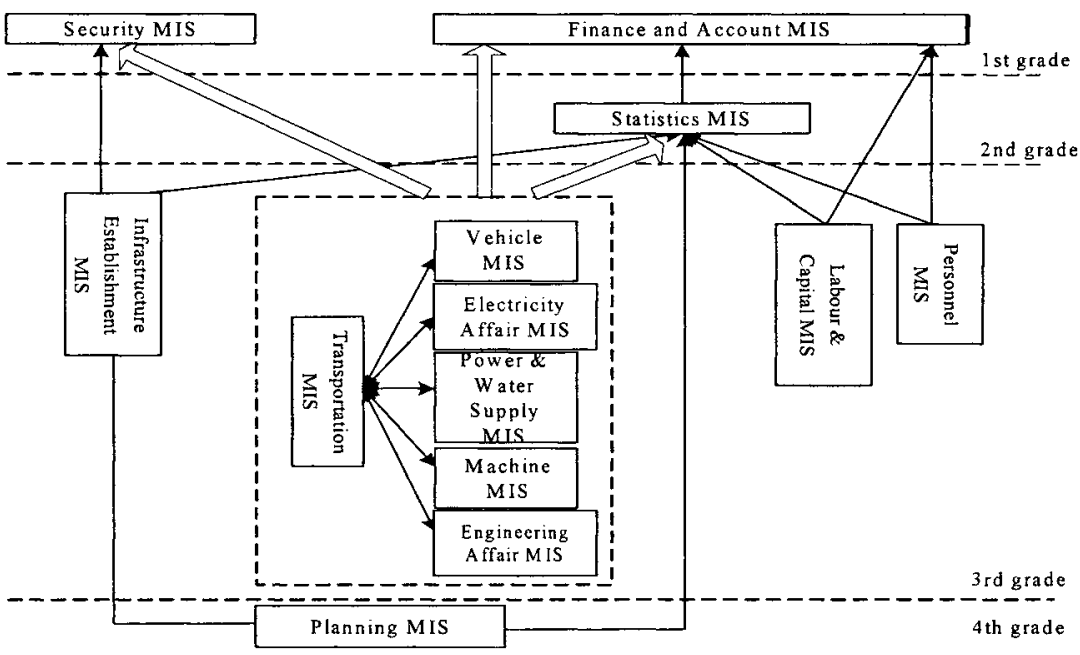

Fig. 2. The multi hierarchy structure of information communication in each operation MIS

The relation and structure of railway MISs have characters as follows: (1)The transport, vehicle, electricity affairs, electricity and water supply, machine affairs and engineering affairs MISs have strong circulation connections. (2)Security and finance MISs are the highest grade in the railway MISs, other operation MISs finally reach them. (3)Personnel MIS and labor and capital MIS are on the same grade with the same higher grade relation. Their positions and functions are relatively 
communicated. (4)Statistics MIS is on the second class, the daily operation information of every MISs finally reach it. (5)Planning MIS does not have MIS reach it and has the lowest grade. Therefore, the integration of railway MISs, should be based on the integration of infrastructure establishment MIS, planning MIS, personnel MIS, labour and capital MIS, statistics MIS, vehicle MIS, electricity affair MIS, power and water supply MIS, machine MIS, engineering affair MIS and transportation MIS, which have strong circulation connection, and be leaded by security MIS, finance and account MIS to make full use of their driving force.

\section{Conclusion}

The information communication relation and intensity among MISs can be measured by the hierarchy analysis method of general structure, separately demonstrating the strong connections between MISs. When planning MISs in a certain industry, develops and integrates MISs with strong connections first. The higher the grade of MIS is, the great prior the overall layout design should be given, for more information from other MISs reach it.

\section{Acknowledgment}

The Special Program for the Preliminary Research of Momentous Fundamental Research of Ministry of Science \& Technology of China(2005CCA03900); The Program for the Preliminary Research of Momentous Fundamental Research of Ministry of Science \& Technology of China(2006CB705504). "An Empirical Study on Chinese E-commerce Development of Transport Industry" (2006RC023), Beijing Jiaotong University science fund, 2006.

\section{References}

1. XUE Huacheng, Management Information System(the 3rd edition), Qinghua university publishing house, Beijing, 1999, pp.263.

2. LI Guogang, LI Baoshan, Management System Engineering, China People University publishing house, Beijing, 1998, pp.107-119.

3. LIU Zhijun, Fulfill the Requirement of Tri-representation, Catch the New Historical Opportunities, Realize the Great-leap-forward Development of China Railway, People's railway, 2003-6-29 (1-3).

4. LI Zhonghao, CHEN Yong, LIU Jun, China's General Situation of the Informationization Establishment in Railway and Transportation Industry in the Year of 2000, Chinese information almanac 2001, China, 2000.

6. ZHANG Guowu, QIAN Dalin, ZHANG Xiuyuan, Traffic and Transportation Planning and Decision-making Support System, China railway publishing house, Beijing 1996, pp.163-168,186. 
7. WANG Linshu, The Opportunity and Challenge Facing Railway Informationization, Traffic and transportation systematical engineering and information, vol.1, no.4, pp.288-293, 2001.

8. Research on Railway Information Share Demand Analysis and Frame Structure, Beijing Jiaotong University Research Report, 2003(unpublished). 